\title{
Transport and Accumulation of Spin Anisotropy
}

\author{
Michael M.E. Baumgärtel, ${ }^{1}$ Michael Hell, ${ }^{1}$ Sourin Das, ${ }^{2,1}$ and Maarten R. Wegewijs ${ }^{1,3}$ \\ ${ }^{1}$ Peter Grünberg Institut, Forschungszentrum Jülich \& JARA Jülich Aachen Research Alliance, D-52425 Jülich, Germany \\ ${ }^{2}$ Department of Physics and Astrophysics, University of Delhi, Delhi 110 007, India \\ ${ }^{3}$ Institute for Theory of Statistical Physics, RWTH Aachen, 52056 Aachen, Germany
}

(Received 29 September 2010; published 16 August 2011)

\begin{abstract}
We show that spin anisotropy can be transferred to an isotropic system by transport of a spinquadrupole moment. We derive the quadrupole moment current and continuity equation and study a spin-valve structure consisting of two ferromagnets coupled to a quantum dot probing an impurity spin. The quadrupole backaction on their coupled spin results in spin torques and anisotropic spin relaxation which do not follow from standard spin-current considerations. We demonstrate the detection of the impurity spin by charge transport and its manipulation by electric fields.
\end{abstract}

PACS numbers: 85.75. $-\mathrm{d}$, 73.63.Kv, 85.35. $-\mathrm{p}$

The field of spintronics is driven by the desire to use the intrinsic dipole moment of the electron, resulting from its spin, as an information carrier. The investigation and design of spintronic devices has made great progress in understanding the accumulation of spin-dipole moments, their manipulation by, e.g., current induced spin torques, and their readout by electrical transport measurements. Recently these studies have been extended to molecular scale quantum dot (QD) devices [1], in particular, the predicted [2] interplay of virtual tunneling, spin polarization and Coulomb interaction has been experimentally demonstrated in spin-valve structures [3]. Furthermore, the importance of intrinsic spin anisotropy, induced by strong spin-orbit interaction, has been demonstrated in several measurements of transport through single magnetic molecules [4] and its various effects have been studied theoretically $[5,6]$. Using various experimental techniques, it was shown that the intrinsic magnetic anisotropy can even be controlled by atomic STM manipulation [6], mechanical straining [7] and reversible charging of the molecule controlled by a gate voltage [8]. The spin anisotropy is of central importance to molecular scale spin manipulation, as it can provide an energy barrier preventing unwanted spin reversal. This has been a key motivation in the field of single-molecule magnetism and proposals for quantum computing with magnetic molecules [9] also rely on spin anisotropy.

Spin anisotropy of a quantum state can be quantified by the average of the quadrupole moment tensor operator

$$
\hat{Q}_{i j}=\frac{1}{2}\left(\hat{S}_{i} \hat{S}_{j}+\hat{S}_{j} \hat{S}_{i}\right)-\frac{1}{3} \hat{\mathbf{S}}^{2} \delta_{i j},
$$

where $\hat{S}_{i}$ is the $i=x, y, z$ component of the spin operator $\hat{\mathbf{S}}$. This operator is nonzero only for spin values $\geq 1$ and traceless. Its components appear in the spin Hamiltonians describing the intrinsic spin anisotropy of magnetic molecules. In this Letter we show that in a QD that by itself is spin isotropic a nonequilibrium spin-quadrupole moment (SQM) can be induced by connecting it to ferromagnets.
We demonstrate that the SQM is a transport quantity that obeys a continuity equation with SQM currents, which can be as important as spin currents. We illustrate this for transport across two ferromagnets contacting a single orbital side-coupled to an impurity spin $1 / 2$ as depicted in Fig. 1. Although electrons have only a spin-dipole moment, they can still transport quadrupole moment: The transport of one of the two dipoles making up a quadrupole moment is shown to result in a net SQM current. Although even simpler spin-valve setups can be found that exhibit nonzero SQM currents in the nonequilibrium stationary state, the system studied here provides the simplest example where a finite SQM can accumulate due to the high-spin $S=1$ in one of the QD charge states. Whenever SQM accumulation occurs, the spin accumulations in the two successive charge states as well as the QD charge are influenced. In this way SQM currents affect the measurable charge current. We show that when we neglect this important quantity the calculated charge current is unphysical: it can be directed opposite to the applied transport bias, even for a collinear spin valve. Our study illustrates that spin anisotropy stored elsewhere (ferromagnets) can be transferred to a place of interest (quantum dot-impurity system), even by single-electron transport. Importantly, this anisotropy, in contrast to the intrinsic anisotropy in molecules $[6-8,10]$, depends on the applied electric fields. Throughout the Letter we set $e=\hbar=k_{\mathrm{B}}=1$ and distinguish between an operator $\hat{A}$ and its average $A=\langle\hat{A}\rangle$ only when needed.

High-spin valve model.-In QDs close to resonance, two adjacent discrete charge states dominate the transport.

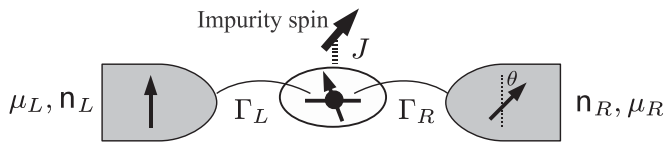

FIG. 1. Quantum dot high-spin valve: noncollinear ferromagnets coupled to an orbital level probing a spin impurity. 
The simplest model Hamiltonian of a QD with nonzero spin in both these charge states [11] is

$$
H=\varepsilon \hat{N}+U \hat{N}_{\uparrow} \hat{N}_{\downarrow}-J \hat{\mathbf{s}} \cdot \hat{\mathbf{s}}^{\prime} .
$$

Here, $\varepsilon$ denotes the energy of an orbital with occupation operators $\hat{N}=\sum_{\sigma} \hat{N}_{\sigma}, \hat{N}_{\sigma}=d_{\sigma}^{\dagger} d_{\sigma}$ with $\sigma=\uparrow, \downarrow$ and spin $\hat{\mathbf{s}}=\frac{1}{2} \sum_{\sigma \sigma^{\prime}} d_{\sigma}^{\dagger} \boldsymbol{\sigma}_{\sigma \sigma^{\prime}} d_{\sigma^{\prime}}$ with Pauli-matrix vector $\boldsymbol{\sigma}$. The electron on the orbital is side coupled to a spin $1 / 2 \hat{\mathbf{s}}^{\prime}$ with isotropic ferromagnetic exchange $J>0$. This may represent, e.g., a QD coupled to a magnetic impurity [12] or a fullerene [13], an asymmetrically gated double QD [14], or even a hyperfine-coupled single nuclear spin in a molecule [15]. Notably, both sign and magnitude of $J$ can be tuned in nanojunctions $[4,16]$ in the few $\mathrm{meV}$ range and $U$ can be several tens of meV. We consider the limit $U \gg J \gg V_{b}$, $T \gg \Gamma_{r}$ where $V_{b}$ is the bias voltage and $\Gamma_{r}$ the tunnel rate, which can be below $0.1 \mathrm{meV}$. Then the electron number $N$ is restricted by Coulomb blockade to 0 or 1 and the $N=1$ singlet state can be neglected due to the strong exchange. Keeping only the ground states of the coupled orbitalimpurity system with spin $1 / 2(N=0)$ and $1(N=1)$, respectively, and energy difference $\epsilon=\varepsilon-J / 4$, we obtain the simplest realization of an isotropic high-spin QD. The orbital is tunnel coupled to noncollinearly polarized ferromagnets (FM), $H_{\mathrm{F}}=\sum_{r k \tau} \epsilon_{r k \tau} c_{r k \tau}^{\dagger} c_{r k \tau}$, with a constant, spin-polarized density of the states $k$ (DOS) $\sum_{k} \delta\left(\epsilon_{r k \tau}-\omega\right) \approx \nu_{r \tau}$ where $\tau=\uparrow, \downarrow$ refers to the spin of the electrons quantized along the polarization axis $\mathbf{n}_{r}$ of the respective electrode $r=L, R$. We let the length of the vector $\mathbf{n}_{r}$ denote the relative polarization of the density of states of the FM: $\left|\mathbf{n}_{r}\right|=\left(\nu_{r \uparrow}-\nu_{r \downarrow}\right) /\left(\nu_{r \uparrow}+\nu_{r \downarrow}\right)$ where $\nu_{r \uparrow}>\nu_{r \downarrow}$ by the definition of $\mathbf{n}_{r}$. The tunnel coupling to the FMs is accounted for by $H_{\mathrm{T}}=\sum_{r k \sigma \tau} t_{r \sigma \tau} d_{\sigma}^{\dagger} c_{r k \tau}+$ H.c., where $\sigma$ refers to the spin of electrons quantized along an axis fixed to the QD (the choice of which drops out of the calculation). The tunneling through junction $r$ is assumed to conserve spin and occurs with a spin-independent amplitude $t_{r}$, thereby setting the tunnel rates $\Gamma_{r}=2 \pi \sum_{\tau} \nu_{r \tau} t_{r}^{2}$. The spin dependence of tunnel amplitude in $H_{T}, t_{r \sigma \tau}=$ $\left\langle\sigma\left|e^{-i \frac{1}{2} \boldsymbol{X}_{r} \cdot \boldsymbol{\sigma}}\right| \tau\right\rangle t_{r}$, arises because we use a different quantization axis in each part of the system. Here, $\chi_{r}=\left|\boldsymbol{\chi}_{r}\right|$ is the angle of rotation about the vector $\boldsymbol{\chi}_{r}$, which maps the QD $z$ axis onto the polarization vector $\mathbf{n}_{r}$. The electrodes are held at temperature $T$ and the transport is controlled (i) by biasing the electrochemical potentials $\mu_{r}= \pm V_{b} / 2$ of the electrodes with $V_{b}$, (ii) by controlling the level position $\epsilon=-V_{g}$ through the gate voltage $V_{g}$, and (iii) by adjusting the relative polarization angle $\theta\left(\mathbf{n}_{L} \cdot \mathbf{n}_{R}=\left|\mathbf{n}_{L}\right|\left|\mathbf{n}_{R}\right| \cos \theta\right)$.

Transport quantities and continuity equations.-The theory we now develop can address the important question how the impurity spin in the above model can be detected by charge transport and controlled by the applied voltages. To understand how charge, spin and SQM can accumulate on the high-spin QD, we first derive the associated current operators and continuity equations. The change of the number operator $\hat{N}$ for electrons localized on the QD is induced by the injected electron particle currents, $\hat{I}_{N}^{r}=$ $-i\left[H_{\mathrm{T}}, \hat{N}^{r}\right]$ where $\hat{N}^{r}=\sum_{k \tau} c_{r k \tau}^{\dagger} c_{r k \tau}$ is the electron number on FM $r: \dot{\hat{N}}=\sum_{r} \hat{I}_{N}^{r}$. This follows from the conservation of the total charge of the system, $\hat{N}^{\text {tot }}=\hat{N}+\sum_{r} \hat{N}^{r}$, in a tunneling process, $\left[H_{T}, \hat{N}^{\text {tot }}\right]=0$, and the conservation of charge on the QD without tunneling, $[H, \hat{N}]=0$. A similar consideration for the spin operators shows that the change in the QD spin $\hat{\mathbf{S}}$ is generated entirely by the spin-conserving tunneling if the QD is spin isotropic, i.e. $[H, \hat{\mathbf{S}}]=0: \dot{\hat{\mathbf{S}}}=\sum_{r} \hat{\mathbf{I}}_{\mathbf{S}}^{r}$ with spin-current operators $\hat{\mathbf{I}}_{\mathbf{S}}^{r}=$ $-i\left[H_{\mathrm{T}}, \hat{\mathbf{S}}^{r}\right]$ and spin operator $\hat{\mathbf{S}}^{r}=\frac{1}{2} \sum_{k \tau \tau^{\prime}} c_{r k \tau}^{\dagger} \boldsymbol{\sigma}_{\tau \tau^{\prime}} c_{r k \tau^{\prime}}$ for FM $r$. The central aspect of the high-spin QD of interest here is that the average of the local quadrupole tensor (1) can become nonzero: the spin triplet state is then anisotropic in addition to spin polarized. This anisotropy accumulates only due to the SQM currents that transport SQM from the spin-anisotropic ferromagnets to the QD:

$$
\dot{\hat{\mathbf{Q}}}=\sum_{r} \hat{\mathbf{I}}_{\mathbf{Q}}^{r}
$$

Here, $\hat{\mathbf{I}}_{\mathbf{Q}}^{r}$ is the SQM current operator associated with electrode $r$,

$$
\hat{\mathbf{I}}_{\mathbf{Q}}^{r}=\frac{1}{2}\left(\hat{\mathbf{I}}_{\mathbf{S}}^{r} \hat{\mathbf{S}}+\hat{\mathbf{S}} \hat{\mathbf{I}}_{\mathbf{S}}^{r}\right)-\left(\frac{1}{3} \hat{\mathbf{I}}_{\mathbf{S}}^{r} \cdot \hat{\mathbf{S}}\right) \hat{\mathbf{I}}+\text { H.c. }
$$

The SQM continuity Eq. (3) derives from the conservation of the total SQM of the system in the tunneling. It equivalently follows from definition (1), using the product rule for the time-derivative, $\frac{d}{d t} \hat{Q}_{i j}=\frac{1}{2}\left(\frac{d \hat{S}_{i}}{d t} \hat{S}_{j}+\hat{S}_{i} \frac{d \hat{S}_{j}}{d t}\right)-$ $\frac{1}{3} \frac{d \hat{\mathbf{S}}}{d t} \hat{\mathbf{S}} \delta_{i j}+(i \leftrightarrow j)$ and the spin continuity equation. The latter form makes explicit that the transport of SQM is enabled by separately transporting the dipoles $\left(\frac{d \hat{S}_{i}}{d t}\right.$ and $\left.\frac{d \hat{S}_{j}}{d t}\right)$ that make up the SQM. Note that the expectation value of the SQM current in (3) bears no simple relation to the spin current since in general $\left\langle\hat{\mathbf{I}}_{\mathbf{S}}^{r} \hat{\mathbf{S}}\right\rangle \neq\left\langle\hat{\mathbf{I}}_{\mathbf{S}}^{r}\right\rangle\langle\hat{\mathbf{S}}\rangle$. To calculate the accumulation of charge, spin and SQM on the QD we need to microscopically derive a theory that describes the QD nonequilibrium state determined by the competition between the charge, spin and SQM currents discussed above.

Kinetic equations. - The time evolution of the (reduced) density operator $p$ of the QD, describing its nonequilibrium state, is determined by the QD Hamiltonian $H$ and a kernel in the kinetic (generalized master) equation. We diagrammatically calculate the time-evolution kernel to the first order in $\Gamma_{r}$ in the framework of the real-time transport theory. To describe the QD high-spin valve in the single-electron tunneling (SET) regime, while consistently accounting for quantum coherence [17], all degrees of freedom need to be considered, including eight nondiagonal elements of the QD density matrix in the eigenbasis of $H$. Without making additional approximations we rewrite the resulting kinetic equations as an exactly 
equivalent set of coupled equations for quantum-statistical averages of a complete set of physical operators in which the density operator can be expanded. For our model these are the charge occupancies $p^{d}, p^{t}\left(p^{d}+p^{t}=1\right)$ of the doublet and triplet state, respectively, $\left(N=\operatorname{Tr} \hat{N} p=p^{t}\right)$, the corresponding spin accumulations $\mathbf{S}^{d}, \mathbf{S}^{t}(\mathbf{S}=\operatorname{Tr} \hat{\mathbf{S}} p=$ $\left.\mathbf{S}^{d}+\mathbf{S}^{t}\right)$ and the triplet $\operatorname{SQM~}_{\mathbf{Q}}^{t}\left(\mathbf{Q}=\operatorname{Tr} \hat{\mathbf{Q}} p=\mathbf{Q}^{t}\right)$. Note that in a QD high-spin valve the spin accumulation vectors $\mathbf{S}^{d}, \mathbf{S}^{t}$ in the two accessible charge states differ in orientation and magnitude and need to be calculated separately. Deferring further technical details to elsewhere, we discuss the physical meaning of the resulting equations in the stationary limit:

$$
\begin{aligned}
0=\dot{p}^{d}=- & 3 \gamma^{+} p^{d}+2 \gamma^{-} p^{t}-2 \boldsymbol{\gamma}^{+} \cdot \mathbf{S}^{d}+2 \boldsymbol{\gamma}^{-} \cdot \mathbf{S}^{t} \\
0=\dot{p}^{t}=3 & \gamma^{+} p^{d}-2 \gamma^{-} p^{t}+2 \boldsymbol{\gamma}^{+} \cdot \mathbf{S}^{d}-2 \boldsymbol{\gamma}^{-} \cdot \mathbf{S}^{t} \\
0=\dot{\mathbf{S}}^{d}=- & -\frac{1}{2} \boldsymbol{\gamma}^{+} p^{d}+\frac{1}{3} \boldsymbol{\gamma}^{-} p^{t}-3 \gamma^{+} \mathbf{S}^{d}+\gamma^{-} \mathbf{S}^{t} \\
& +\mathbf{S}^{d} \times \boldsymbol{\beta}+2 \mathbf{Q}^{t} \cdot \gamma^{-} \\
0=\dot{\mathbf{S}}^{t}= & \frac{4}{2} \boldsymbol{\gamma}^{+} p^{d}-\frac{4}{3} \boldsymbol{\gamma}^{-} p^{t}+4 \gamma^{+} \mathbf{S}^{d}-2 \gamma^{-} \mathbf{S}^{t} \\
& +\mathbf{S}^{t} \times \boldsymbol{\beta}-2 \mathbf{Q}^{t} \cdot \gamma^{-} \\
0=\dot{\mathbf{Q}}^{t}= & {\left[\frac{4}{2}\left(\mathbf{S}^{d} \boldsymbol{\gamma}^{+}+\boldsymbol{\gamma}^{+} \mathbf{S}^{d}\right)-\frac{4}{3}\left(\mathbf{S}^{d} \cdot \boldsymbol{\gamma}^{+}\right) \mathbf{1}\right] } \\
& -\left[\frac{1}{2}\left(\mathbf{S}^{t} \boldsymbol{\gamma}^{-}+\boldsymbol{\gamma}^{-} \mathbf{S}^{t}\right)-\frac{1}{3}\left(\mathbf{S}^{t} \cdot \boldsymbol{\gamma}^{-}\right) \mathbf{1}\right] \\
& -2 \gamma^{-} \mathbf{Q}^{t}+\mathbf{Q}^{t} \times \boldsymbol{\beta}-\boldsymbol{\beta} \times \mathbf{Q}^{t}
\end{aligned}
$$

Here, $\gamma^{ \pm}=\sum_{r} \gamma_{r}^{ \pm}$, where $\gamma_{r}^{ \pm}=\frac{1}{2} \Gamma_{r} f_{r}^{ \pm}(\epsilon)$ is the rate for single charge tunneling in (out) of the QD through junction $r$, denoting the Fermi distribution for electrons (holes) by $f_{r}^{ \pm}(\epsilon)=\left(e^{ \pm\left(\epsilon-\mu_{r}\right) / T}+1\right)^{-1}$. Similarly, boldfaced vectors $\boldsymbol{\gamma}^{ \pm}=\sum_{r} \boldsymbol{\gamma}_{r}^{ \pm}$and $\boldsymbol{\gamma}_{r}^{ \pm}=\gamma_{r}^{ \pm} \mathbf{n}_{r}$ denote the corresponding rates of tunneling of spin, polarized along $\mathbf{n}_{r}$, through junction $r$. Finally, $\boldsymbol{\beta}=\sum_{r} \boldsymbol{\beta}_{r}$ is an effective magnetic field with contributions from each electrode $r: \boldsymbol{\beta}_{r}=$ $\frac{1}{2} \Gamma_{r} \operatorname{Re} \int_{-D}^{D} \frac{d \omega}{\pi} \frac{f_{r}^{+}(\omega)}{\omega-\epsilon+i 0} \mathbf{n}_{r}$, where the cutoff needs to be set to $D \sim U$ since we exclude the $N=2$ charge state of the model. This field represents the spin splitting induced on the QD by coherent virtual electron tunneling processes into the spin-polarized electrode $r$ and relies on the nonzero value of the Coulomb charging energy $U$ [2]. The magnitude $\left|\boldsymbol{\beta}_{\mathbf{r}}\right|$ of these exchange fields is electrically tunable [3], with a peak at $\epsilon=\mu_{r}$ and logarithmic tails. The kinetic equations incorporate the conservation of probability, $p^{d}+p^{t}=1$, and the tracelessness of the SQM tensor, $\operatorname{tr} \mathbf{Q}^{t}=\sum_{i} Q_{i i}^{t}=0$. The stationary charge current through junction $r$ can be calculated similarly:

$$
I_{N}^{r}=3 \gamma_{r}^{+} p^{d}-2 \gamma_{r}^{-} p^{t}+2 \boldsymbol{\gamma}_{r}^{+} \cdot \mathbf{S}^{d}-2 \boldsymbol{\gamma}_{r}^{-} \cdot \mathbf{S}^{t} .
$$

The charge continuity equation is satisfied: $\dot{N}=\dot{p}^{t}=$ $-\dot{p}^{d}=\sum_{r} I_{N}^{r}=0$ in the stationary limit.

Because of the spin polarization of the electrodes, the charge occupancies (5) and (6) and the current (10) couple to both charge-specific spins, but are not directly influenced by the SQM. Equations (7) and (8) show that these spins couple back to the charge occupancies, and suffer isotropic spin relaxation $\left(\propto-\gamma^{+} \mathbf{S}^{d}\right.$ and $\propto-\gamma^{-} \mathbf{S}^{t}$, respectively). In addition there is a transfer of spin polarization from one charge state to the other $\left(\propto \gamma^{-} \mathbf{S}^{t}\right.$ and $\propto \gamma^{+} \mathbf{S}^{d}$, respectively). The next-to-last term represents a torque on the charge-specific spin due to the exchange field $\boldsymbol{\beta}$.

A central result of this Letter is that the spin accumulated in each charge state also couples to the SQM accumulated in the triplet state through the last term $\pm 2 \mathbf{Q}^{t} \cdot \boldsymbol{\gamma}^{-}$in (7) and (8). The opposite signs of these terms indicate that a nonzero SQM tends to make the spin polarizations in the two charge states noncollinear. The accumulation of SQM is described by the kinetic Eq. (9): the net injection (first two terms, in dyadic notation $(\mathbf{a b})_{i j}=a_{i} b_{j}$ ) competes with the isotropic relaxation (third term). The last two terms, $\left(\mathbf{Q}^{t} \times \boldsymbol{\beta}\right)_{i j}=\epsilon_{j k l} Q_{i k}^{t} \beta_{l}$ and $\left(\boldsymbol{\beta} \times \mathbf{Q}^{t}\right)_{i j}=\epsilon_{i k l} \beta_{k} Q_{l j}^{t}$, incorporate a torque exerted on the SQM by the exchange field $\boldsymbol{\beta}$. This finite SQM results in a back-action on the spin when substituted into the righthand side of (7) and (8). Importantly, these backaction terms are comparable to the other terms in (7) and (8): solving Eq. (9) for $\mathbf{Q}^{t}$ in terms of $\mathbf{S}^{d}$ and $\mathbf{S}^{t}$ we obtain

$$
2 \mathbf{Q}^{t} \cdot \boldsymbol{\gamma}^{-}=\sum_{\lambda=d, t}\left(\mathbf{R}^{\lambda} \cdot \mathbf{S}^{\lambda}+\mathbf{S}^{\lambda} \times \boldsymbol{\beta}^{\lambda}\right) .
$$

In Eq. (11) the symmetric tensors $\mathbf{R}^{\lambda}$ make the spin relaxation and the spin transfer anisotropic. The remaining terms have the form of a spin torque involving new, chargestate specific exchange fields $\boldsymbol{\beta}^{\lambda}$, which can be shown to be noncollinear with the standard exchange field $\boldsymbol{\beta}$. These have the twofold effect of modifying the existing spin torque term in Eqs. (7) and (8), $\boldsymbol{\beta} \rightarrow \boldsymbol{\beta} \pm \boldsymbol{\beta}^{d, t}$, and adding a torque that involves the spin from the other charge state. The latter thus represents a transfer of spin torque between the two charge states. In contrast to the standard exchange field $\boldsymbol{\beta}$, which is of a purely coherent origin, both $\boldsymbol{\beta}^{\lambda}$ and $\mathbf{R}^{\lambda}$ arise from a complex interplay of dissipative and coherent processes: their (lengthy) expressions contain both the transition rates $\boldsymbol{\gamma}^{ \pm}$and the exchange field $\boldsymbol{\beta}$. We emphasize that the above spin relaxations and spin torques in (11) cannot be understood as arising from spin (or charge) currents: for a correct description of the spin dynamics the transport of SQM must be accounted for. By its effect on the spin, the SQM also acts back on the charge occupancies and the measurable charge current cf. Eq. (10). From an exhaustive study of all parameter regimes of the model including arbitrary noncollinear magnetizations we find that in general the SQM affects the charge transport considerably whenever significant 
spin accumulation occurs, i.e., in the regime of polarizations $\left|\mathbf{n}_{L}\right|,\left|\mathbf{n}_{R}\right| \geqslant 0.5$ of interest for spintronics applications. If one neglects the transport and accumulation of SQM in Eqs. (5)-(9) one can obtain an unphysical, large particle current running opposite to the voltage bias direction for nearly all relative polarization angles $\theta$, for instance when the drain electrode is most strongly spinpolarized and tunnel coupled $\left(\left|\mathbf{n}_{L}\right|<\left|\mathbf{n}_{R}\right| \approx 1, \Gamma_{L}<\Gamma_{R}\right)$. In this case the components of the quadrupole accumulation are of the order of the maximal achievable value $2 / 3$ (in units $\hbar^{2}$ ). We now return to the questions raised for the studied model.

Impurity spin detection and control.-When similar FMs $\left(\left|\mathbf{n}_{L}\right|=\left|\mathbf{n}_{R}\right|\right)$ with nearly antiparallel polarizations $(\theta \approx \pi)$ are asymmetrically tunnel coupled $\left(\Gamma_{L}>\Gamma_{R}\right)$ to the QD, the presence of the side-coupled impurity spin can be detected by nonlinear transport measurements. Figures 2(a)-2(c) show that a sharp, anomalous current peak occurs in the thermally broadened regime where usually the SET current through the QD switches on. The peak height depends nonmonotonically on $V_{b}$ and its position depends nonlinearly on $V_{g}$. The occurrence of such a current peak for opposite polarities of the bias and gate voltage (relative to the degeneracy point) indicates the presence of ferromagnetic coupling of the orbital to an impurity spin, resulting in nonzero total spin in both accessible charge states. Indeed, a calculation that ignores the impurity spin completely, only shows the current peak for forward bias. Figures 2(d) and 2(e) show that in both cases the current peak directly measures a significant precession of the spin accumulation on the QD. This strong precession in a narrow range of voltages is possible due the asymmetric tunneling coupling. At the bias and gate voltage where the effect is maximal the magnitude of the field $\boldsymbol{\beta}_{R}(\boldsymbol{\epsilon})$ originating from the weakly coupled FM is resonantly enhanced $\left(\left|\epsilon-\mu_{R}\right| \approx T\right)$ and matches the magnitude of $\boldsymbol{\beta}_{L}(\boldsymbol{\epsilon})$ from the strongly coupled FM, which is off resonance $\left(\left|\epsilon-\mu_{L}\right| \gg T\right)$. For $\theta \sim \pi$ the exchange field $\boldsymbol{\beta}$ is a sum of the two nearly antiparallel, equal-length vectors and is therefore perpendicular to the spin, which accumulates in one of the QD charge states opposite to either $\mathbf{n}_{R}$ or $\mathbf{n}_{L}$ for $\mu_{L} \gtrless \mu_{R}$; see Figs. 2(d) and 2(e). Although this exchange field is small, it does, however, cause a large spin precession over an angle $\sim \pi$ due to the onset of Coulomb blockade, which suppresses the spin relaxation. When the weakly coupled ferromagnet is tuned off resonance with $V_{g}$ or $V_{b},\left|\epsilon-\mu_{R}\right| \gg T$, this precession is switched off. The correct description of this electrically controlled spin resonance relies on both the spin-transfer terms (cf. blue curve) as well as the SQM induced backaction on the spin (cf. red curve).

Conclusion.-We have illustrated the importance of nonequilibrium transport of spin anisotropy and its interplay with charge and spin-dipole accumulation for the simplest type of high-spin QD. This analysis can be generalized to QD spin-valves with higher spins $S-\frac{1}{2}>\frac{1}{2}$ and $S>1$ in its subsequent charge states for which the transport of spinmultipole moments of ranks up to $2 S$ needs to be included. This shows that spintronics requires more than just charge and spin currents for theoretical predictions of device operation, e.g., the electrical detection and manipulation of spin [6,11]. Moreover, this insight brings new possibilities in view. For instance, the design of experimental setups that support pure SQM currents (not accompanied by spin currents) is of interest for creating spin reversal barriers similar to those in magnetic adatoms [10] and molecules [8].
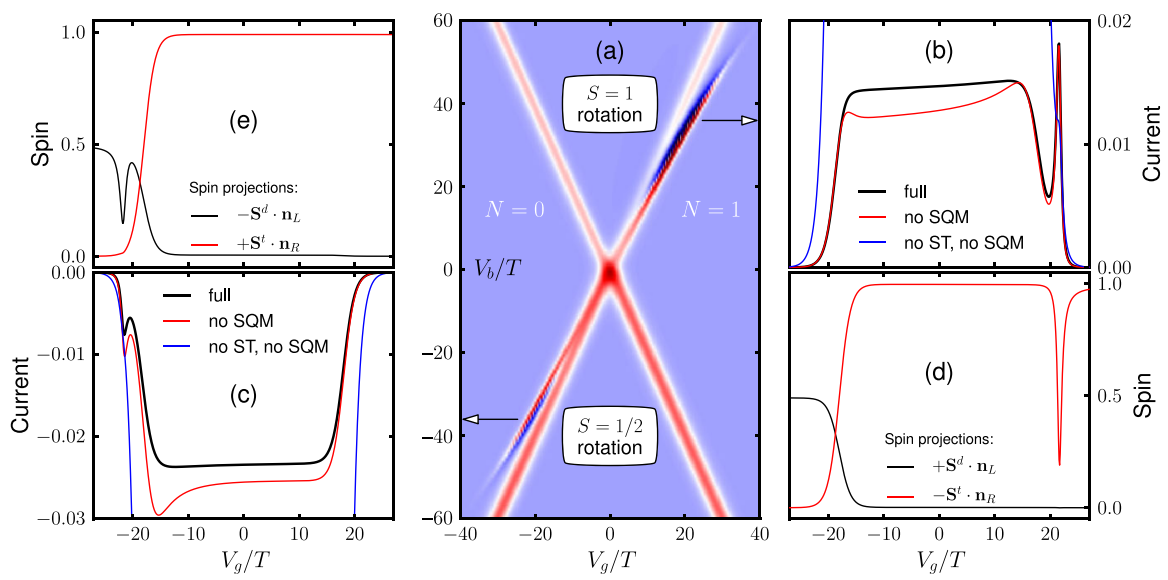

FIG. 2 (color online). (a) Stability diagram $d I / d V_{b}$ vs $V_{g}, V_{b}\left(\right.$ red $=$ positive, dark blue $=$ negative). Parameters: $\Gamma_{\mathrm{L}}=2 \Gamma_{\mathrm{R}}=$ $0.2 T$ and $D=U=500 T$ in units of the temperature $T$. The high polarization $\left|\mathbf{n}_{L}\right|=\left|\mathbf{n}_{R}\right|=0.99$ allows all effects to be clearly illustrated, lower values only result in a rescaling of the current plateaus. Notably, a slight noncollinearity of the magnetization, $\theta=0.99 \pi$, has a distinct and significant effect. (b),(c): $I$ vs $V_{g}$ for $V_{b}= \pm 36 T$ normalized to the maximal current $I_{0}=\left(\left(3 \Gamma_{L, R}\right)^{-1}+\right.$ $\left.\left(2 \Gamma_{R, L}\right)^{-1}\right)^{-1}$ achievable for $V_{b} \gtrless 0$ and parallel polarizations $(\theta=0)$. Black: full result, red: neglecting the SQM, blue: additionally neglecting the spin-transfer (ST) terms in Eq. (7) and (8). (d),(e): Spin projections $\pm \mathbf{S}^{d} \cdot \mathbf{n}_{L}$ and $\mp \mathbf{S}^{t} \cdot \mathbf{n}_{R}$ affecting the current (10) vs $V_{g}$ corresponding to (b) and (c), respectively. 
However, in contrast to the latter no intrinsic anisotropy is required. Moreover, the SQM accumulation can both compete with as well as reinforce the quadrupolar effect induced by additional intrinsic anisotropy of the exchange interaction with the impurity [18]. Our results may also be of interest for quantum information processing: SQM currents in combination with spin currents may assist in preparing a spin-1 quantum state of a qutrit (two exchange coupled qubits) by time-dependent transport. Our work has shown the sensitivity of charge transport to spin-anisotropy currents and a next challenge is the design of setups that can directly detect SQM tensor currents.

We acknowledge stimulating discussions with B. Sothmann, J. König, and S. Andergassen. and the financial support from NanoSci-ERA and DFG (FOR 912).

[1] S. Sahoo et al., Nature Phys. 1, 99 (2005).

[2] J. König and J. Martinek, Phys. Rev. Lett. 90, 166602 (2003).

[3] A. N. Pasupathy et al., Science 306, 86 (2004); J. R. Hauptmann et al., Nature Phys. 4, 373 (2008).

[4] H. B. Heersche et al., Phys. Rev. Lett. 96, 206801 (2006); E. A. Osorio et al., Nano Lett. 10, 105 (2010).
[5] F. Elste and C. Timm, Phys. Rev. B 73, 235305 (2006); G. Gonzalez and M. N. Leuenberger, Phys. Rev. Lett. 98, 256804 (2007); M. Misiorny, I. Weymann, and J. Barnaś, Phys. Rev. B 79, 224420 (2009).

[6] B. Sothmann and J. König, Phys. Rev. B 82, 245319 (2010); A. F. Otte et al., Nature Phys. 4, 847 (2008).

[7] J. J. Parks et al., Science 328, 1370 (2010).

[8] A. S. Zyazin et al., Nano Lett. 10, 3307 (2010).

[9] M. N. Leuenberger and D. Loss, Nature (London) 410, 789 (2001).

[10] C. F. Hirjibehedin, C. P. Lutz, and A. J. Heinrich, Science 312, 1021 (2006); C. F. Hirjibehedin et al., Science 317, 1199 (2007); A. F. Otte et al., Phys. Rev. Lett. 103, 107203 (2009).

[11] G. Kiesslich et al., Appl. Phys. Lett. 95, 152104 (2009).

[12] H. B. Heersche et al., Phys. Rev. Lett. 96, 017205 (2006).

[13] J.E. Grose et al., Nature Mater. 7, 884 (2008).

[14] K. Kikoin and Y. Avishai, Phys. Rev. B 65, 115329 (2002).

[15] N. Ishikawa et al., J. Am. Chem. Soc. 127, 3650 (2005).

[16] N. Roch et al., Phys. Rev. Lett. 103, 197202 (2009).

[17] M. Braun, J. Konig, and J. Martinek, Phys. Rev. B 70, 195345 (2004); A. Donarini, G. Begemann, and M. Grifoni, Nano Lett. 9, 2897 (2009).

[18] J. Fernández-Rossier and R. Aguado, Phys. Rev. Lett. 98, 106805 (2007). 